\title{
Signal processing and frequency-dependent associative memory based on nanoswitches
}

\author{
Javier Cervera, ${ }^{1}$ Patricio Ramírez, ${ }^{2}$ and Salvador Mafé ${ }^{1, a)}$ \\ ${ }_{1}^{1}$ Facultat de Física, Universitat de València, E-46100 Burjassot, Spain \\ ${ }^{2}$ Departament de Física Aplicada, Universitat Politècnica de València, E-46022 València, Spain
}

(Received 25 April 2008; accepted 11 September 2008; published online 31 October 2008)

\begin{abstract}
A signal processing concept based on nanoscale switches whose conductance can be tuned by an external stimulus between two (ON and OFF) states is proposed and analyzed theoretically. The building block of the system is formed by a metal nanoparticle linked to two electrodes by an organic ligand and a molecular switch. When we apply an alternating potential to the system of the same frequency as the periodic variation between the ON and OFF states induced on the switch, the net charge delivered by the system exhibits a sharp resonance. This resonance can be used to process an external signal by selectively extracting the weight of the different harmonics. In addition, a frequency-dependent associative memory that exploits the resonance property is also demonstrated. The general properties of the signal processing concept that are required for practical implementation are analyzed for different temperatures, times, conductance ratios, applied potentials, and noise conditions. (C) 2008 American Institute of Physics. [DOI: 10.1063/1.3006817]
\end{abstract}

\section{INTRODUCTION}

Nanostructures have attracted great interest as a future solution to the continuous miniaturization of electronics. The small size of molecules can allow high packing densities together with rich functional properties, ${ }^{1-4}$ although they present challenges in their design and in the interfacing with current electronics. ${ }^{3}$ One of the building blocks of molecular electronics is nanoscale switches, formed by nanostructures that can vary their conductance between two states because of an external stimulus. This can be accomplished by the change between two conformational or redox states of a nanostructure (e.g., a bipyridinium molecule; see Ref. 5 and references therein) induced by a light pulse, ${ }^{6-9}$ an electrochemical reaction, ${ }^{10-12}$ or a mixed photochemical/ electrochemical process. ${ }^{13,14}$ In addition, monolayerprotected metallic nanoparticles ${ }^{15-20}$ can be employed to optimize the electron transfer between the electrodes and the switching molecule. ${ }^{21}$

The experimental characterization and modeling of particular nanoscale systems are receiving much attention. However, it is also necessary to design general schemes that could perform information storage and processing tasks using nanostructures. ${ }^{3,22,23}$ In this work, we extend significantly a preliminary communication ${ }^{22}$ and explore theoretically the use of nanoscale switches to implement a signal processing capable of decoding an external signal. The processing is based on the sharp resonance of the net charge delivered by the system that occurs when the frequency of the applied alternating potential is equal to that of the periodic variation induced on the conductance of the switch (see Fig. 1). This allows decoding a global input signal by extracting the weight of the different harmonics. In addition, we study the use of nanoscale switches to implement a sort of frequencydependent associative memory that retrieves the stored pat-

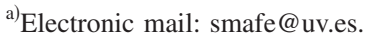

tern most similar to a given input pattern. ${ }^{23}$ In this case, we introduce the stored patterns using a continuous modulation of the switch conductance and define bit coincidence/ mismatch by combining frequency and phase shifts. Rather than consider with detail a particular nanosystem, we analyze the general properties of the proposed signal processing concept making use of a highly idealized model. Because an expanding list of chemically, physically, and electrochemically switchable nanosystems have experimentally been

(external stimulus)
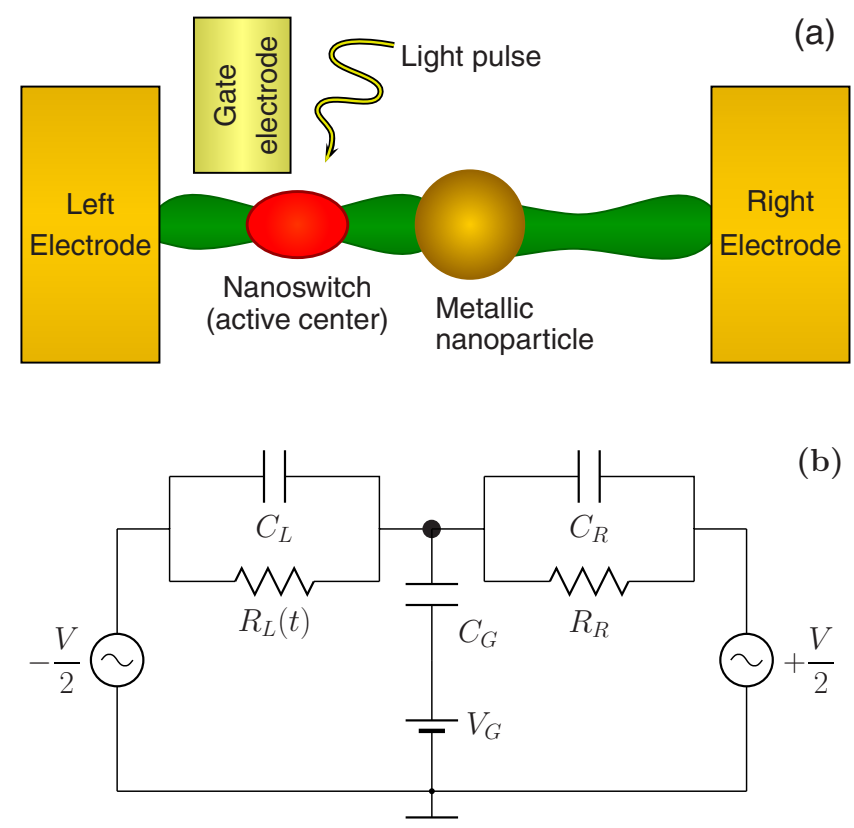

FIG. 1. (Color online) (a) Schematic view of the central nanoparticle and the two ligands that link it to the electrodes (Ref. 22). One of the ligands is a nanoswitch whose conductance can be changed between the values $G_{M}$ and $G_{m}$ by using an external stimulus (the gate electrode or a light pulse in the figure). (b) The equivalent circuit of the system. 
demonstrated, ${ }^{5}$ this study can stimulate future particular realizations by showing the essential characteristics of the concept.

\section{MODEL}

The system of Fig. 1 is composed of a metallic nanoparticle linked to the electrodes by two organic ligands. ${ }^{22}$ Electron transport occurs by tunneling between the electrodes and the nanoparticle, which is modulated by the ligands. The left ligand has an active center whose state can be modified externally so that its tunneling conductance $G_{L}$ changes between the values $G_{M}$ and $G_{m}$. The right ligand has a constant conductance $G_{R} \gg G_{M}, G_{m}$, which is much smaller than the quantum conductance $G_{0}=2 e^{2} / h=77.4 \mu \mathrm{S}$, where $e$ is the elementary charge and $h$ is the Planck constant. The physical model employed was described previously ${ }^{22}$ and it is summarized briefly for the sake of completeness. We assume that the conductance of the left ligand can be modulated periodically with a period $\tau$ :

$$
G_{L}(t)=\left\{\begin{array}{ll}
G_{m}, & 0<t<\tau / 2 \\
G_{M}, & \tau / 2<t<\tau
\end{array} .\right.
$$

When the modulation frequency $\nu_{0}=1 / \tau$ satisfies the condition $h \nu_{0}<k T$, the tunneling of the electrons between the nanoparticle and the electrodes can be described by the rates $^{24}$

$$
\begin{aligned}
\Gamma_{L, n}^{ \pm}(t) & =\frac{G_{L}(t)}{e^{2}} \frac{-\Delta E_{L, n}^{ \pm}}{1-\exp \left(\Delta E_{L, n}^{ \pm} / k T\right)}, \\
\Gamma_{R, n}^{ \pm}(t) & =\frac{G_{R}}{e^{2}} \frac{-\Delta E_{R, n}^{ \pm}}{1-\exp \left(\Delta E_{R, n}^{ \pm} / k T\right)},
\end{aligned}
$$

where $\Gamma_{L, n}^{ \pm}$and $\Gamma_{R, n}^{ \pm}$are the tunneling rates of the left $(L)$ and right $(R)$ electrodes for electron transitions between the electrodes and the nanoparticle from left to right $(-)$ and from right to left $(+) . \Delta E_{L, n}^{ \pm}$and $\Delta E_{R, n}^{ \pm}$are the change in electrostatic energy between the electronic occupation states $n$ and $n+1$ of the nanoparticle. Because the conductances of the ligands are much smaller than $G_{0}$, electrons are localized inside the nanoparticle. This allows to calculate $\Delta E_{L, n}^{ \pm}$and $\Delta E_{R, n}^{ \pm}$using the orthodox theory [see Fig. 1(b)]: ${ }^{20,24}$

$$
\begin{aligned}
& \Delta E_{L, n}^{ \pm}(t)=\mp \frac{e^{2}}{C_{\Sigma}}\left[\frac{1}{2}+n-\frac{C_{G} V_{G}}{e}-\frac{\left(C_{R}+C_{G} / 2\right) V}{e}\right], \\
& \Delta E_{R, n}^{ \pm}(t)= \pm \frac{e^{2}}{C_{\Sigma}}\left[\frac{1}{2}+n-\frac{C_{G} V_{G}}{e}+\frac{\left(C_{L}+C_{G} / 2\right) V}{e}\right],
\end{aligned}
$$

where $C_{G}$ and $V_{G}$ are the capacitance and the potential of a secondary gate electrode, which injects a charge $Q_{G}=C_{G} V_{G}$ on the nanoparticle. The use of this gate electrode is usual, ${ }^{25-27}$ but it is not essential for the system performance at high temperature (see Appendix). The total capacitance of the system is $C_{\Sigma}=C_{L}+C_{R}+C_{G}$. The occupation state of the nanoparticle can be obtained using the master equation: ${ }^{24,25}$

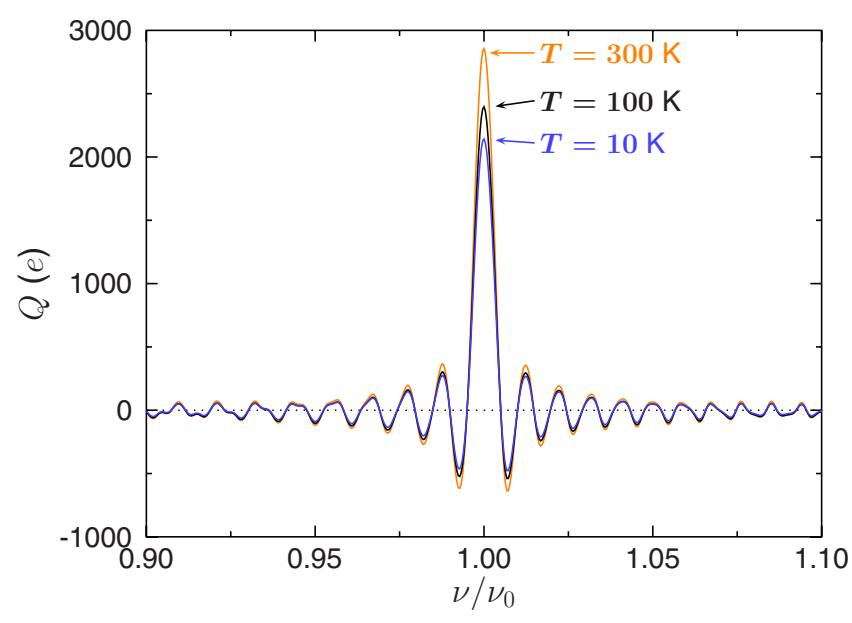

FIG. 2. (Color online) The net charge delivered by the system of Fig. 1 as a function of the dimensionless frequency of the alternating potential for the temperatures in the figure and $t_{0}=1 \mathrm{~ms}$.

$$
\begin{aligned}
\frac{d P_{n}}{d t}= & \left(\Gamma_{L, n-1}^{-}+\Gamma_{R, n-1}^{+}\right) P_{n-1}+\left(\Gamma_{L, n}^{+}+\Gamma_{R, n}^{-}\right) P_{n+1} \\
& -\left(\Gamma_{L, n-1}^{+}+\Gamma_{R, n-1}^{-}+\Gamma_{L, n}^{-}+\Gamma_{R, n}^{+}\right) P_{n},
\end{aligned}
$$

where $P_{n}(t)$ is the probability of the occupation state $n$. Initially, no electrons have been transferred to the nanoparticle, and thus $P_{0}(0)=1$ and $P_{i}(0)=0(i>0)$. The time evolution of the probabilities $P_{n}(t)$ allow to calculate the current as

$$
I(t)=-e \sum_{n}\left[\Gamma_{L, n}^{-} P_{n}(t)-\Gamma_{L, n}^{+} P_{n+1}(t)\right] .
$$

From this current, we can obtain the net charge delivered at time $t_{0}$ as

$$
Q=\int_{0}^{t_{0}} I(t) d t
$$

We use the net charge delivered to assess the feasibility and performance of the system because $Q$ can be detected by using output capacitors of relatively large capacitances as interfaces between the nanoswitch system and a complementary metal-oxide semiconductor (CMOS) circuit (these capacitors would give experimentally observable timedependent potentials ${ }^{23}$ ).

Current noise can severely limit the performance of the nanosystems. ${ }^{20,28}$ We estimate current fluctuations by using the full counting statistics approach, which gives an evaluation of the probability distribution function of the electrons transferred to a given electrode. ${ }^{28,29}$ The second moment of this distribution corresponds to the fluctuations in the net charge delivered $\left\langle(Q-\langle Q\rangle)^{2}\right\rangle$ from which we estimate the error in $Q$ as $\Delta^{*} Q=\sqrt{\left\langle(Q-\langle Q\rangle)^{2}\right\rangle}$.

\section{RESULTS AND DISCUSSION}

\section{A. Signal processing}

Figure 2 shows the typical results obtained ${ }^{22}$ for the net charge delivered by the system as a function of the frequency of the applied alternating potential $V(t)=V_{0} \sin (2 \pi \nu t)$. The curves are parametric in the temperature with $t_{0}=1 \mathrm{~ms}$ and 
$V_{0}=1 \mathrm{mV}$. The capacitances of the ligands are $C_{L}=C_{R}$ $=1 \mathrm{aF}$. The conductances are $G_{R}=100 \mathrm{nS}$ for the right ligand and $G_{M}=10 \mathrm{nS}$ and $G_{m}=5 \mathrm{nS}$ for the ON and OFF states of the left ligand [see Eq. (1)]. The capacitance and potential of the secondary gate electrode are $C_{G}=4 \mathrm{aF}$ and $V_{G}=20 \mathrm{mV}$. These values are typical of nanostructures. ${ }^{19,20,22,23,30}$ The frequency of the conductance switching function of Eq. (1) is $\nu_{0}=10^{5} \mathrm{~s}^{-1}$. To obtain an efficient modulation of $G_{L}$, we must introduce a frequency $\nu_{0}$ much lower than the characteristic relaxation frequency for the change in conductance of the left ligand. We need to consider also the characteristic sampling frequency bandwidth of the detector, which may limit the frequencies used in the conductance modulation in practical operation. In any case, the system can operate also at much lower frequencies because the switching period is set externally. The drawback of a lower frequency is the longer the operational time $t_{0}$, albeit with a lower noise ratio, as it will be shown later.

Our scheme makes use of the concept of resonance discussed by Pistolesi and Fazio. ${ }^{25}$ However, it is significantly different from previous studies with oscillating electromechanical nanostructures. $^{25-27}$ The main feature of the net charge delivered by the system of Fig. 1 is the sharp resonance $^{25}$ at $\nu=\nu_{0}$ (see Fig. 2). The resonance peak depends on the left conductance ratio $G_{m} / G_{M}$, being more marked for large differences between $G_{m}$ and $G_{M}$. This peak increases with the time $t_{0}$ and the applied potential $V_{0}$. The tunneling rates are also affected by the temperature [see Eqs. (2) and (3)]: at very low temperatures $k T \ll|\Delta E|$, only those tunneling transitions that are energetically favorable $(\Delta E$ $<0$ ) occur, but the probability of unfavorable transitions becomes significant as the temperature increases. This gives an increase in the electron tunneling as the unfavorable transitions are added to the energetically favorable ones (the latter are not strongly affected by the increase in temperature). The above effect leads to a higher net delivery at the resonance peak, except for very high temperatures because these would have the effect of leveling the transition rates, thus decreasing the resonance peak. The increase in temperature also yields a noise increase that should be carefully considered because the system of Fig. 1 is the building block for the signal processing device.

The resonance in the net charge delivered permits to decode a pattern codified as a global signal. ${ }^{22}$ This is done by extracting the state of the different harmonics using the arrangement of seven nanoswitches shown schematically in Fig. 3. A switching function of characteristic frequency $\nu_{i}^{0}$ is applied to the left conductance of every building block $i$ [see Eq. (1)] and the system is fed with the global input potential

$$
V(t)=V_{0} \sum_{i=1}^{7} a_{i} \sin \left(2 \pi \nu_{i}^{0} t\right)
$$

codifying the input signal. The input vector $\boldsymbol{a}$ $=\left(a_{1}, a_{2}, \ldots, a_{7}\right)$ stores the bits of a seven-segment number. In the example of Fig. $3, \boldsymbol{a}=(0,1,1,0,0,1,1)$, which corresponds to number $4 .^{22}$ The input potential $V(t)$ induces a resonant response only on those nanoswitches where $a_{i}=1$ (see Fig. 2) and therefore the selected nanoswitches will de-

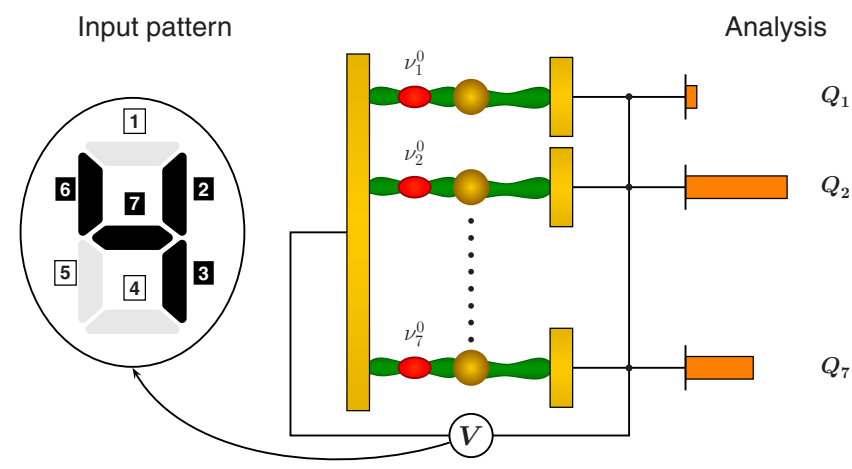

FIG. 3. (Color online) Scheme of the signal processing system (Ref. 22) with seven nanoswitches similar to that in Fig. 1. The nanoswitches represent the bits of the seven-segment number shown in the figure. In particular, the input number 4 is described by the vector $\boldsymbol{a}=(0,1,1,0,0,1,1)$ in the input potential $V(t)=V_{0} \sum_{i=1}^{7} a_{i} \sin \left(2 \pi \nu_{i}^{0} t\right)$. The individual external stimulus (the gate electrode or the light pulse in Fig. 1) on each nanoswitch is omitted for clarity.

liver a high net charge $Q$. On the contrary, the nanoswitches with $a_{i}=0$ will deliver a low net charge. A threshold charge can be established to identify (beyond the uncertainty introduced by the noise) those nanoswitches, which give a resonant response. This permits to decode the input signal by extracting the state of the different harmonics, retrieving thus the value of vector $\boldsymbol{a}$.

Figure 4 corresponds to the analysis ${ }^{22}$ of the potential $V(t)$ for the input number 4 (see Fig. 3) and the same parameters of Fig. 2, with $T=300 \mathrm{~K}$ in Figs. 4(b)-4(d). The nanoswitches differ in the frequency of the switching function only. The switching frequency of nanoswitch 1 is $\nu_{1}^{0}$ $=10^{5} \mathrm{~s}^{-1}$ and those of the rest are obtained as $\nu_{i}^{0}=2 \nu_{i-1}^{0}, i$ $>1$. Figure 4 shows that the system is able to process efficiently the input signal, retrieving which segments are "black" and which are "white" (see Fig. 3). Remarkably, the system of nanoswitches of Fig. 3 can retrieve correctly the input number for a wide range of experimental parameters (temperature, potential, conductance ratio, and time). Figure 4(a) considers the dependence of the system performance with temperature. While the increase in temperature leads to an increase in the net charge delivered, as it allows transitions with $\Delta E>0$, it causes also a noise increase (see the error bars of Fig. 4). This may prevent the correct retrieval of the state of the segments in Fig. 3. However, changes in the values of the parameters such as an increase in the applied potential $V_{0}$, a larger difference between $G_{L}$ and $G_{R}$, or a longer operational time $t_{0}$ will restore the correct retrieval. ${ }^{22}$ In particular, there is a minimum potential that allows retrieving correctly the input number beyond the noise uncertainty [see Fig. 4(b)]. Although the fluctuations increase in absolute value with $V_{0}$, their relative values with respect to the net charge decrease, and this allows the correct signal processing. Figure 4(c) shows the necessity of small enough conductance ratios $G_{m} / G_{M}$ to process efficiently the input signal. Remarkably, Fig. 4(c) predicts that a variation between $G_{m}$ and $G_{M}$ as low as $50 \%$ suffices for the present case (the lower $G_{m} / G_{M}$, the better the performance). The conductance ratio affects also the operational range of the other system parameters. 

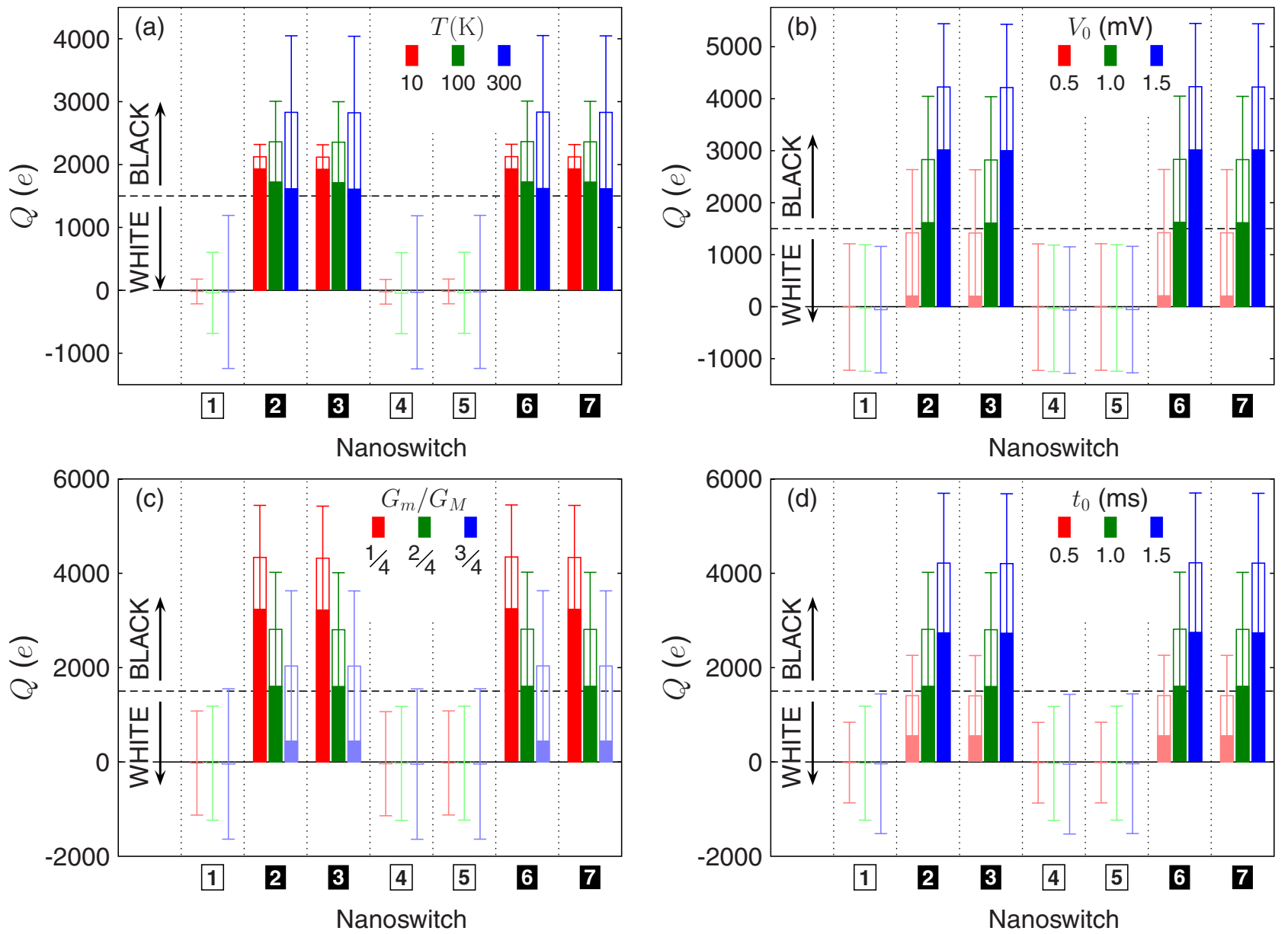

FIG. 4. (Color online) Signal processing of an input signal that codifies input number 4. The arrangement of Fig. 3 is considered for different values of (a) temperature, (b) potential, (c) conductance ratio, and (d) time. (b)-(d) correspond to $T=300 \mathrm{~K}$. The dark colored bars are retrieved as black segments and the light colored bars are retrieved as white segments (see Fig. 3). The uncertainty $\Delta^{*} Q$ in the charge delivered is given by the error bars.

The proposed design makes use of the net charge delivered by each nanoswitch to retrieve the input signal (number). This cumulative property is expected to be robust with respect to fluctuations. However, this also means that the time needed for correct retrieval is much higher than the period of the switching function. Indeed, in Fig. 4(d) the operational time for the system is of the order of a milisecond, whereas the characteristic signal periods are 100 times lower (the retrieval time needs to be much higher than the period of the switching function to overcome the limitations introduced by noise). The characteristic frequencies $\nu_{i}^{0}$ would determine the typical times involved in practical operation.

The signal processing is based on the resonance that occurs when the frequency $\nu$ of one of the harmonics in the input signal matches the frequency $\nu_{i}^{0}$ of the switching function of one of the nanoswitches in the system. Because $\nu_{i}^{0}$ is set externally, the system can be adapted to work on a prescribed frequency range. Figure 5 shows the signal processing of an input signal that codifies input number 4 for $t_{0}$ $=0.1 \mathrm{~s}$ and the temperatures $T=100$ (left bar), 200 (central bar), and $300 \mathrm{~K}$ (right bar). We consider the same system of Fig. 4 but decreasing the switching frequency of nanoswitch 1 to $\nu_{1}^{0}=500 \mathrm{~s}^{-1}$, with $\nu_{i}^{0}=2 \nu_{i-1}^{0}$. This gives the highest frequency $\nu_{7}^{0}=32 \mathrm{kHz}$. As shown in Fig. 5 , the system is also able to retrieve the state of the segments forming the input number using this new set of lower frequencies. The large operational time is a consequence of the lower frequencies used and implies a higher net charge delivered by the nanoswitches as well as a lower noise (in relative terms).

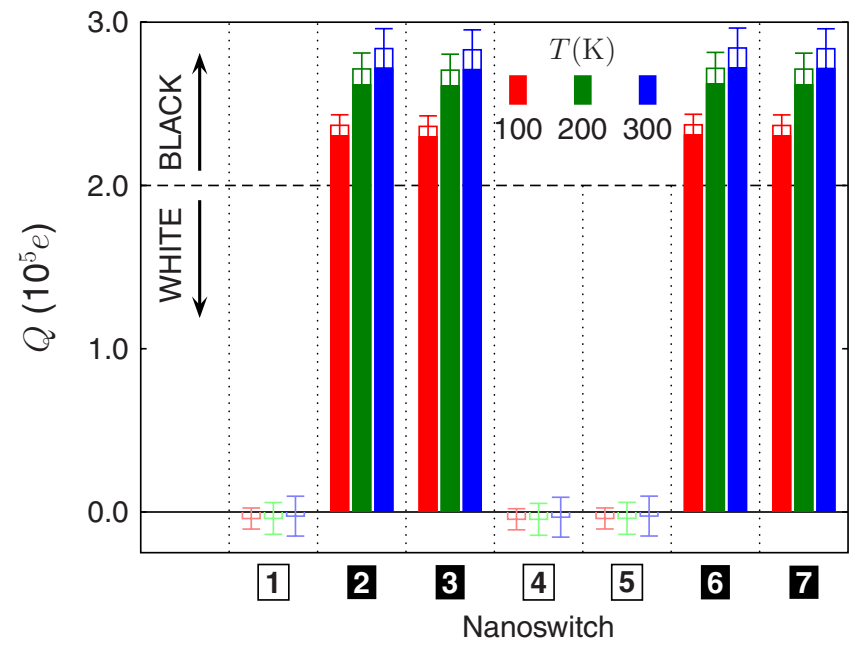

FIG. 5. (Color online) Signal processing at low frequency of an input signal that codifies input number 4 using the arrangement of Fig. 3 for the temperatures $T=100$ (left bar), 200 (central bar), and $300 \mathrm{~K}$ (right bar). The frequencies of the nanoswitches are $\nu_{i}^{0}=2 \nu_{i-1}^{0}$, with $\nu_{1}^{0}=500 \mathrm{~Hz}$ and the operational time is $t_{0}=0.1 \mathrm{~s}$. 


\section{B. Associative memory}

A resonance in frequency similar to that shown in Fig. 2 can also be obtained when the conductance of the switch does not change abruptly between $G_{m}$ and $G_{M}$, but follows the modulation function

$$
G_{L}(t)=\frac{G_{M}+G_{m}}{2}\left[1+\frac{G_{M}-G_{m}}{G_{M}+G_{m}} \sin \left(2 \pi \nu_{0} t\right)\right]
$$

instead of Eq. (1). This resonance can also be exploited to implement signal processing using the design of Fig. 3. In addition, the continuous variation in $G_{L}$ between $G_{m}$ and $G_{M}$ offers the possibility of implementing a sort of frequencydependent associative memory. In this case, patterns are stored in the left conductance modulation, and the system allows for the comparison and retrieval of that pattern in the modulation that is most similar to the input pattern.

Consider the subset of the seven segment numbers of Fig. 3 formed by numbers 0,1 , and 2 only. We have then $N=3$ patterns stored using $M=7$ bits. The number of bits that differs between two of the above patterns (the so called Hamming distance $)^{23}$ is at least three (see Fig. 3). Pattern recognition will rely on the net charge delivered by the nanoswitches, following a scheme analogous to that used in a previous design based on the static conductance of the building blocks. ${ }^{23}$ To this end, we need to devise a method that associates a high value of the net charge with every bit coincidence and a low value of the net charge with every bit mismatch.

If the conductance of the left ligand changes continuously between $G_{m}$ and $G_{M}$, we can store a given pattern (number) in the modulation of the conductance of a given nanoswitch as follows. Every pattern $j=0,1,2$ is defined by choosing the functional form for the modulation of the left ligand as

$$
\begin{aligned}
G_{L}^{j}(t)= & \frac{G_{M}+G_{m}}{2}+\frac{G_{M}-G_{m}}{14} \sum_{i=1}^{7}\left[a_{i}^{j} \sin \left(2 \pi \nu_{i}^{0} t\right)\right. \\
& \left.+\left(1-a_{i}^{j}\right) \cos \left(2 \pi \nu_{i}^{0} t\right)\right],
\end{aligned}
$$

where $a_{i}^{j}=1$ if segment $i$ of pattern $j$ is black and $a_{i}^{j}=0$ if it is white (see Fig. 3). In this way, the three patterns are stored in the three modulation functions of Eq. (11), one pattern per nanoswitch (thus only three nanoswitches are now needed). The input pattern is introduced now through the applied potential

$$
V(t)=V_{0} \sum_{i=1}^{7}\left[a_{i}^{\mathrm{in}} \sin \left(2 \pi \nu_{i}^{0} t\right)+\left(1-a_{i}^{\mathrm{in}}\right) \cos \left(2 \pi \nu_{i}^{0} t\right)\right],
$$

where $a_{i}^{\text {in }}=1$ if segment $i$ of the input pattern is black and $a_{i}^{\text {in }}=0$ if it is white. Note that bit coincidence/mismatch is defined by combining frequency and phase shifts in Eqs. (11) and (12). The separation between the different segments of each number is made through the frequency shift. For each segment $i$, bit coincidence occurs when the respective terms of the input [Eq. (12)] and the stored [in the nanoswitch modulation; see Eq. (11)] patterns are in phase, whereas bit

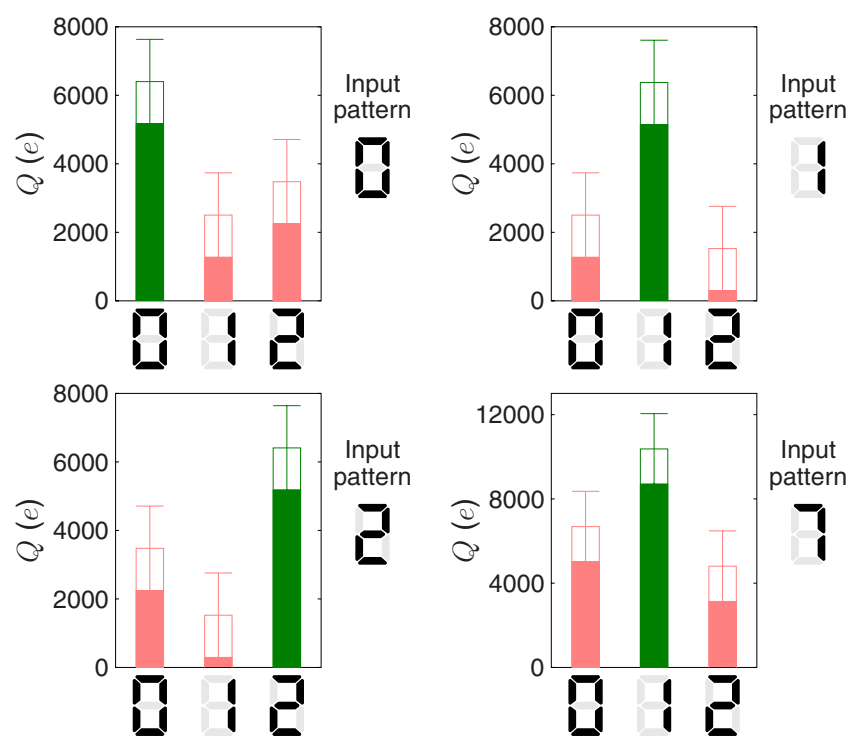

FIG. 6. (Color online) Pattern retrieval for input numbers 0, 1, 2, and 7. Note that we have only one nanoswitch per stored pattern. Patterns are stored in the time modulation of the left conductance [see Eq. (11)]. The charge $Q$ delivered by each nanoswitch during $t_{0}=1 \mathrm{~ms}$ (input numbers 0 , 1 , and 2) and $2 \mathrm{~ms}$ (input number 7) is the property used to retrieve the stored pattern most similar to the input pattern (dark colored bar). The error bars correspond to the uncertainty in the charge $\Delta^{*} Q$.

mismatch occurs when these terms have a phase shift in $\pm \pi / 2$.

Figure 6 shows the net charge delivered by the three nanoswitches corresponding to the three patterns 0,1 , and 2 for the input numbers $0,1,2$, and 7 [the last number is not stored in the modulation of Eq. (11)]. The operational time is $t_{0}=1 \mathrm{~ms}$ for input numbers 0,1 , and 2, whereas $t_{0}=2 \mathrm{~ms}$ for input number 7. All three nanoswitches are equal. The capacitances of the ligands are $C_{L}=C_{R}=1 \mathrm{aF}$. The conductances are $G_{R}=100 \mathrm{nS}$ for the right ligand and $G_{M}=10 \mathrm{nS}$ and $G_{m}=5 \mathrm{nS}$ for the left ligand. The capacitance and potential of the secondary gate electrode are $C_{G}=4 \mathrm{aF}$ and $V_{G}=20 \mathrm{mV}$, respectively. The amplitude of the applied potential is $V_{0}=1.5 \mathrm{mV}$. The seven frequencies that correspond to the seven segments (bits) are $\nu_{i}^{0}=2 \nu_{i-1}^{0}$, with $\nu_{1}^{0}=10^{5} \mathrm{~s}^{-1}$, and the temperature is $T=300 \mathrm{~K}$. The results of Fig. 6 are obtained now with Eqs. (2)-(8), (11), and (12).

Figure 6 shows that the net charge delivered by each nanoswitch increases with the number of bit coincidences between the input pattern and the stored pattern. This is readily explained if we consider that the tunneling rates between the left electrode and the nanoparticle [and then the current $I(t)]$ are proportional to $G_{L}(t) V(t)$. This product is formed by terms such as $\sin \left(2 \pi \nu_{i}^{0} t\right) \sin \left(2 \pi \nu_{j}^{0} t\right)$, $\sin \left(2 \pi \nu_{i}^{0} t\right) \cos \left(2 \pi \nu_{j}^{0} t\right)$, and $\cos \left(2 \pi \nu_{i}^{0} t\right) \cos \left(2 \pi \nu_{j}^{0} t\right)$. Upon time integration (to obtain $Q$ ), only those terms with $\nu_{i}^{0}=\nu_{j}^{0}$ and a null phase shift (two sines or two cosines) contribute significantly to the net charge delivered. Because these terms are associated with bit coincidences, pattern recognition can be achieved using the charge $Q$. Note that this scheme works also when the input pattern is not one of the stored patterns [see Fig. 6(d)]. In this case, the higher $Q$ is obtained for the stored pattern (1), which is most similar to the input pattern (7). The retrieval is now obtained by increasing slightly the 

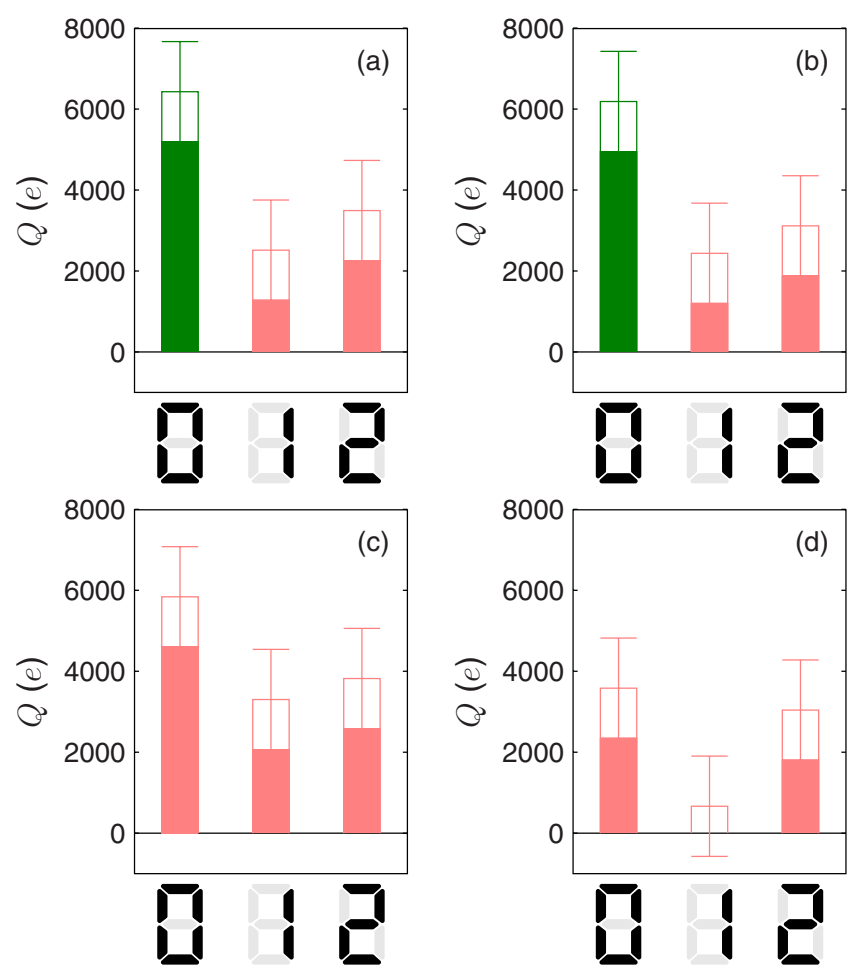

FIG. 7. (Color online) Pattern retrieval for input pattern 0. Patterns are stored in the time modulation of Eq. (13) and the phase noise amplitude is (a) $\Delta \phi_{0}=0$, (b) $\pi / 8$, (c) $\pi / 4$, and (d) $\pi / 2$.

time $t_{0}$. Clearly, the system performance as an associative memory will improve by increasing the number of bits associated with every pattern.

The error bars for the pattern recognition of Fig. 6 were evaluated assuming (exact) phase matches for bit coincidence and (exact) $\pm \pi / 2$ phase shifts for bit mismatch. However, a certain amount of phase noise between the input potential $V(t)$ and the modulation of the conductance $G_{L}(t)$ is to be expected in practical operation. To estimate the influence of phase noise, we assume that the left ligand conductance of nanoswitch $j$ has the form

$$
\begin{aligned}
G_{L}^{j}(t)= & \frac{G_{M}+G_{m}}{2}+\frac{G_{M}-G_{m}}{14} \sum_{i=1}^{7}\left[a_{i}^{j} \sin \left(2 \pi \nu_{i}^{0} t+\delta \phi_{i}^{a}\right)\right. \\
& \left.+\left(1-a_{i}^{j}\right) \cos \left(2 \pi \nu_{i}^{0} t+\delta \phi_{i}^{b}\right)\right]
\end{aligned}
$$

where the phase noise terms $\delta \phi_{i}^{a}$ and $\delta \phi_{i}^{b}$ are random numbers in the interval $\left[-\Delta \phi_{0}, \Delta \phi_{0}\right]$, and $\Delta \phi_{0}$ is the noise amplitude. $V(t)$ is still given by Eq. (12), and thus $\delta \phi_{i}^{a}$ and $\delta \phi_{i}^{b}$ are the (constant) phase shifts between $G_{L}(t)$ and $V(t)$ caused by the noise. Figure 7 shows the pattern retrieval for input pattern 0 with the noise amplitudes (a) 0 , (b) $\pi / 8$, (c) $\pi / 4$, and (d) $\pi / 2$. The parameters used are those of Figs 6(a)-6(c). The results show the general trends of Fig. 6 despite the fact that the phase shifts vary because of the use of random numbers. The simulations predict that the system is moderately robust to phase noise; the pattern is retrieved correctly when the phase noise amplitude is up to $25 \%$ of the phase shift that defines bit coincidence/mismatch. Indeed, because of the sharp resonance in frequency, frequency noise is more critical for the performance than phase noise. As the noise amplitude increases, pattern retrieval becomes poorer. Eventually, the associative memory renders useless when the phase noise masks the difference between bit coincidence and mismatch [Fig. 7(d)], as it could be expected.

\section{CONCLUSIONS}

We have designed and analyzed theoretically a signal processing scheme based on the electronic transference through a metallic nanoparticle linked to two electrodes by organic ligands. One of the ligands presents an active center whose conductance can be modulated externally between two (ON and OFF) states. If the conductance of the ligand is varied periodically and a time dependent input potential is applied to the electrodes, the net charge delivered by the system will show a resonance in frequency. This charge can be monitored by using output capacitors with large capacitances as interfaces between the system and a CMOS circuit. As shown in a preliminary communication, ${ }^{22}$ the resonance can be exploited to analyze a signal formed by a vector of bits codified in frequency as a Fourier series. The system is able to process efficiently the signal and retrieve the vector of bits correctly for different system parameters (temperature, potential, ratio of conductances, and time; see also Appendix). In addition, using a continuous variation for the ligand conductance between the two states, a frequencydependent associative memory scheme has been demonstrated. Patterns are stored in the time modulation of the nanoswitch conductances and the input pattern is introduced in the time dependent potential applied between the electrodes. Bit coincidence and mismatch are defined using combined frequency and phase shifts. Pattern recognition is based on the net charge delivered by each nanoswitch, which increases with the number of bit coincidences. In each case, rather than consider with detail a particular experimental system, we analyze the general properties of the signal processing scheme making use of a simple model that shows the essential characteristics required for practical implementation.

\section{ACKNOWLEDGMENTS}

Financial support from the European Commission (Project DYNAMO, Project No. FP6-028669-2, and program New and Emerging Science and Technology) is acknowledged.

\section{APPENDIX}

The above results were obtained with a secondary gate electrode ${ }^{25-27}$ characterized by the capacitance $C_{G}=4 \mathrm{aF}$ and the applied potential $V_{G}=20 \mathrm{mV}$. We show in this Appendix that the proposed scheme could work efficiently under different conditions and that the effect of the secondary gate electrode is not critical. In its absence, the system retains the resonance in frequency, and both signal processing and associative memory schemes can still be implemented at high temperatures. This is shown in Figs. 8-10 obtained with the gate capacitance $C_{G}=0$ in Eqs. (4) and (5).

Figure 8 shows the net charge delivered by the system for $t_{0}=1 \mathrm{~ms}$ as a function of the frequency of the alternating 


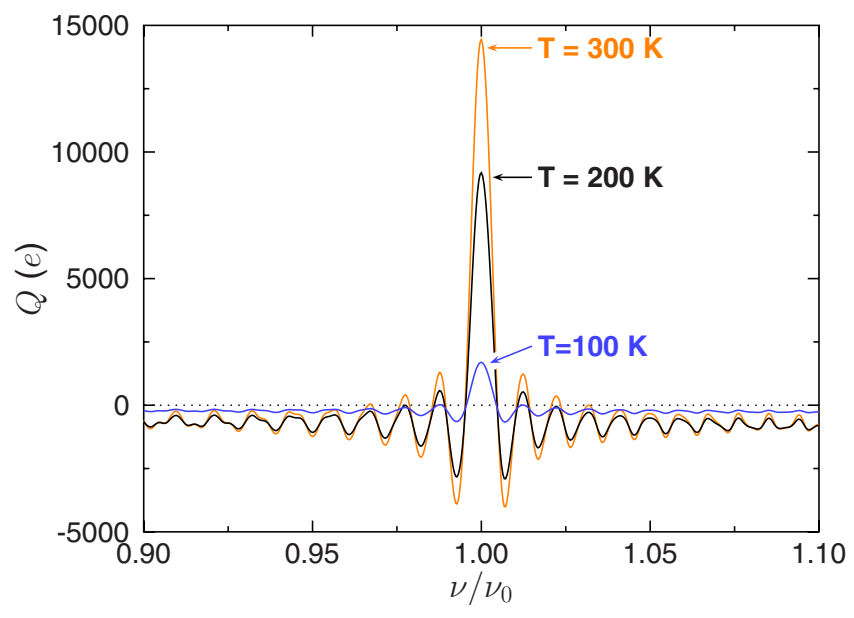

FIG. 8. (Color online) Net charge delivered by the system of Fig. 1 for $t_{0}$ $=1 \mathrm{~ms}$ as a function of the frequency of the alternating potential for different temperatures in the absence of the secondary gate electrode.

potential at different temperatures. The capacitances of the ligands are $C_{L}=C_{R}=1 \mathrm{aF}$, and their conductances $G_{R}$ $=100 \mathrm{nS}$ for the right ligand and $G_{M}=10 \mathrm{nS}$ and $G_{m}$ $=5 \mathrm{nS}$ for the ON and OFF states of the left ligand. The applied potential is $V(t)=V_{0} \sin (2 \pi \nu t)$, with $V_{0}=10 \mathrm{mV}$. The frequency of the conductance switching function is $\nu_{0}$ $=10^{5} \mathrm{~s}^{-1}$. Again, the main feature is the resonance in frequency, especially for high temperatures (compare Fig. 8 with Fig. 2) because for low temperatures the Coulomb blockade effect practically suppresses the electron transitions $\left(\Gamma_{L}^{-}\right.$and $\left.\Gamma_{R}^{+}\right)$from the electrodes to the nanoparticle $(\Delta E>0$ in these cases). It is only with the increase in temperature that the thermal energy can activate the electron transitions with $\Delta E>0$, so that a finite current flows across the system. To increase the current at low temperatures, we need to overtake Coulomb blockade. This can be made by increasing the applied voltage or by using a secondary gate electrode to change the potential of the nanoparticle (see Fig. 2).

Figure 9 shows the signal processing of number 4 , codified into the global potential of Eq. (9), using the scheme of Fig. 3. The values of the characteristic parameters are those

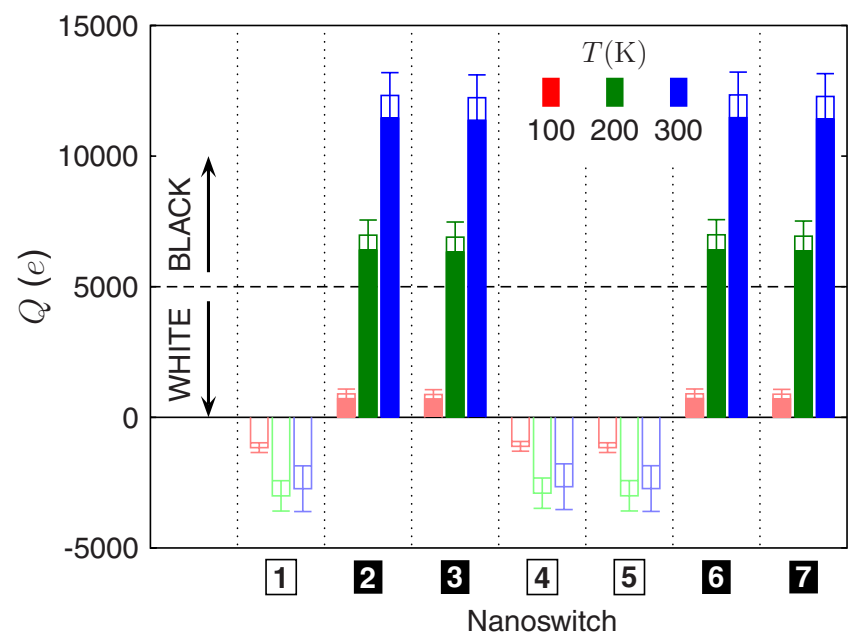

FIG. 9. (Color online) Signal processing using the arrangement of Fig. 3 for input number 4, with $T=100$ (left bar), 200 (central bar), and $300 \mathrm{~K}$ (right bar), in the absence of the secondary gate electrode.

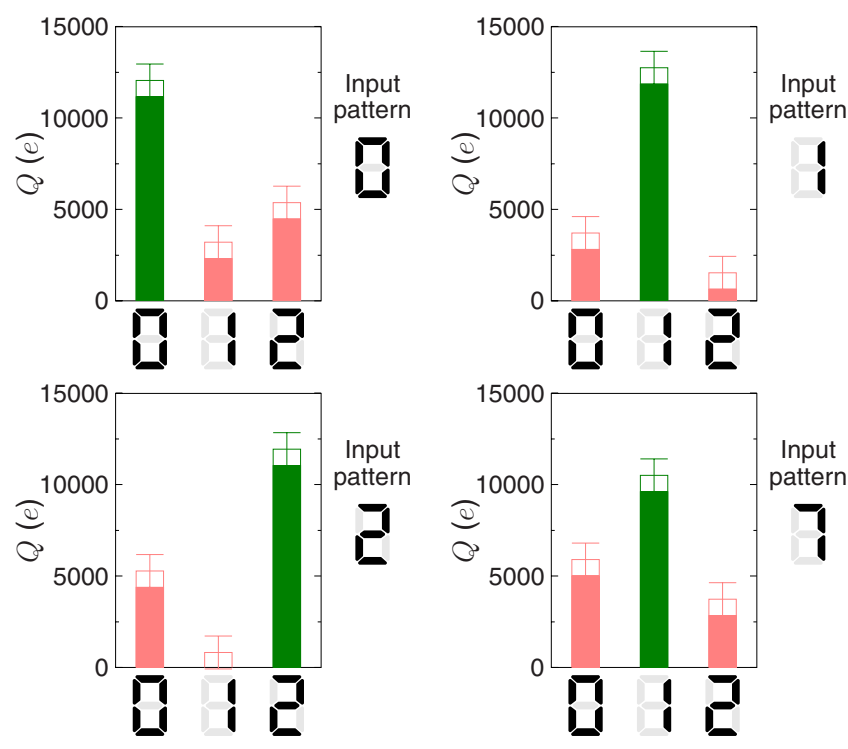

FIG. 10. (Color online) Pattern retrieval for input numbers 0, 1, 2, and 7 in the absence of the secondary gate electrode. The charge $Q$ delivered by each nanoswitch during $t_{0}=1 \mathrm{~ms}$ is the property used to retrieve the stored pattern most similar to the input pattern (dark colored bar).

of Fig. 8 and the switching frequency of the nanoswitch $i$ is $\nu_{i}^{0}=2 \nu_{i-1}^{0}$, with $\nu_{1}^{0}=10^{5} \mathrm{~s}^{-1}$. The retrieval of the segment state improves with temperature (see Fig. 8). The negative value of charge delivered by the nonselected nanoswitches is caused by the fact that $G_{R}>G_{L}(t)$ [the charge would be positive for $\left.G_{R}<G_{L}(t)\right]$. The system is still able to work efficiently without a second gate electrode, which could simplify the experimental realization.

Figure 10 shows the associative memory performance when the secondary gate is absent. The stored pattern is codified in the time modulation of the left ligand conductance [see Eq. (11)] and the input pattern is codified in the applied potential [see Eq. (12)]. The operational time is $t_{0}=1 \mathrm{~ms}$ and the temperature is $T=300 \mathrm{~K}$. All three nanoswitches storing the three patterns 0,1 , and 2 are equal. The capacitances of the ligands are $C_{L}=C_{R}=1 \mathrm{aF}$. The conductances are $G_{R}$ $=55 \mathrm{nS}$ for the right ligand and $G_{M}=55 \mathrm{nS}$ and $G_{m}$ $=27.5 \mathrm{nS}$ for the left ligand. We have used similar values for $G_{R}$ and $G_{L}$ to minimize the effect of the background current (which gives the negative $Q$ observed for nonselected nanoswitches in Fig. 9). The frequencies of the segments are given by $\nu_{i}^{0}=2 \nu_{i-1}^{0}$, with $\nu_{1}^{0}=10^{5} \mathrm{~s}^{-1}$. The amplitude of the applied potential is $V_{0}=10 \mathrm{mV}$. As in Fig. 6, the net charge delivered is significantly higher for the pattern most similar to the input pattern.

${ }^{1}$ Y. Yang, J. Ouyang, L. Ma, R. Tseng, and C.-H. Chu, Adv. Funct. Mater. 16, 1001 (2006).

${ }^{2}$ M. P. Blencowe, Contemp. Phys. 46, 249 (2005).

${ }^{3}$ N. Weibel, S. Grunder, and M. Mayor, Org. Biomol. Chem. 5, 2343 (2007).

${ }^{4}$ D. Chen, G. Wang, and J. Li, J. Phys. Chem. C 111, 2351 (2007).

${ }^{5}$ D. Feldheim, Nature (London) 408, 45 (2000).

${ }^{6}$ D. G. Walter, D. J. Campbell, and C. A. Mirkin, J. Phys. Chem. B 103, 402 (1999).

${ }^{7}$ T. Kudernac, S. J. van der Molen, B. J. van Wees, and B. L. Jeringa, Chem. Commun. (Cambridge) 2006, 3597.

${ }^{8}$ P. Ahonen, T. Laaksonen, D. J. Schiffrin, and K. Kontturi, Phys. Chem. Chem. Phys. 9, 4898 (2007). 
${ }^{9}$ Y. Tanaka, A. Inagaki, and M. Akita, Chem. Commun. (Cambridge) 2007, 1169.

${ }^{10}$ D. I. Gittins, D. Bethell, D. J. Schiffrin, and R. J. Nichols, Nature (London) 408, 67 (2000).

${ }^{11}$ B. Xu, X. Xiao, X. Yang, L. Zang, and N. Tao, J. Am. Chem. Soc. 127, 2386 (2005).

${ }^{12}$ E. H. van Dijk, D. J. T. Myles, M. H. van der Veen, and J. C. Hummelen, Org. Lett. 8, 2333 (2006).

${ }^{13}$ J. Areephong, W. R. Browne, N. Katsonis, and B. L. Feringa, Chem. Commun. (Cambridge) 2006, 3930.

${ }^{14}$ R. Baron, A. Onopriyenko, E. Katz, O. Lioubashevski, I. Willner, S. Wang, and H. Tian, Chem. Commun. (Cambridge) 2006, 2147.

${ }^{15}$ M. Brust, M. Walker, D. Bethell, D. J. Schiffrin, and R. Whyman, J. Chem. Soc., Chem. Commun. 1994, 801.

${ }^{16}$ S. Chen, R. S. Ingram, M. J. Hostetler, J. J. Pietron, R. W. Murray, T. G. Schaaff, J. T. Khoury, M. M. Alvarez, and R. L. Whetten, Science 280 , 2098 (1998).

${ }^{17}$ B. M. Quinn, P. Liljeroth, V. Ruiz, T. Laaksonen, and K. Kontturi, J. Am. Chem. Soc. 125, 6644 (2003).
${ }^{18}$ N. K. Chaki, B. Kakade, K. P. Vijayamohanan, P. Singh, and C. V. Dharmadhikari, Phys. Chem. Chem. Phys. 8, 1837 (2006).

${ }^{19}$ V. García-Morales and S. Mafé, J. Phys. Chem. C 111, 7242 (2007).

${ }^{20}$ J. Cervera and S. Mafé, Chem. Phys. Lett. 451, 257 (2008).

${ }^{21}$ Y. Yamanoi and H. Nishihara, Chem. Commun. (Cambridge) 2007, 3983.

${ }^{22}$ J. Cervera and S. Mafé, "Metallic nanoparticles linked to molecular switches as signal processing devices," J. Nanosci. Nanotechnol. (in press).

${ }^{23}$ S. Mafé, J. A. Manzanares, and J. Cervera, J. Phys. Chem. C 112, 1663 (2008).

${ }^{24}$ K. Likharev, Proc. IEEE 87, 606 (1999).

${ }^{25}$ F. Pistolesi and R. Fazio, Phys. Rev. Lett. 94, 036806 (2005).

${ }^{26}$ A. Erbe, C. Weiss, W. Zwerger, and R. H. Blick, Phys. Rev. Lett. 87, 096106 (2001).

${ }^{27}$ V. Sazonova, Y. Yaish, H. Üstünel, D. Roundy, T. A. Arias, and P. L. McEuen, Nature (London) 431, 284 (2004).

${ }^{28}$ D. A. Bagrets and Y. V. Nazarov, Phys. Rev. B 67, 085316 (2003).

${ }^{29}$ F. Pistolesi, Phys. Rev. B 69, 245409 (2004).

${ }^{30}$ D. J. Wold and C. D. Frisbie, J. Am. Chem. Soc. 122, 2970 (2000). 